\title{
Hemangiopericytoma of the Posterior Fossa: A Case Report and Review of the Literature
}

\author{
II Seo, Yun-Suk Kim, Ho-Sang Kim, Jeong-Ho Kim, Myung-Ki Lee \\ Department of Neurosurgery, Maryknoll Medical Center, Busan, Korea
}

Received March 31, 2013

Revised April 10, 2013

Accepted July 1, 2013

Correspondence

Yun-Suk Kim

Department of Neurosurgery,

Maryknoll Medical Center,

121 Junggu-ro, Jung-gu,

Busan 600-730, Korea

Tel: +82-51-461-2456

Fax: +82-51-466-6164

E-mail: yunsuk.kim@gmail.com

\begin{abstract}
Intracranial hemangiopericytoma is unusual, and those occurring in the posterior fossa is extremely rare; we report such a rare case of hemangiopericytoma of the posterior fossa. The radiologic findings and gross characteristics of hemangiopericytomas are sometimes quite similar to those of meningiomas. Although extremely rare, the operator should be aware of the existence of this disorder to dexterously manage the aggressive nature and high vascular tendency of hemangiopericytomas. The radiological features and histological findings in this case are discussed in this study.
\end{abstract}

Key Words Hemangiopericytoma; Posterior fossa; Meningioma.

\section{INTRODUCTION}

Intracranial hemangiopericytoma (HPC) has been reported to account for 2 to $4 \%$ of meningeal tumors and less than $1 \%$ of all intracranial tumors [1]. Primary intracranial HPC in the posterior fossa has occurred rarely and reports are also seldom encountered [2,3]. The rarity of HPC and its controversial histological classification result in its frequent misdiagnosis and thus make the treatment quite challenging [2]. Furthermore, according to other reports, the long-term biological and clinical behavior of HPC differs from those of meningiomas, revealing local recurrence rates as high as $91 \%$ and a 15 -year risk of distant metastasis nearly $70 \%$ after surgery alone [3]. A surgeon may well experience an unexpected surgical situation due to the highly developed vascularity of HPCs, when the preoperative diagnosis is inaccurate. We describe a rare case of an HPC occurring in the posterior fossa, which was difficult to distinguish from a meningioma in terms of clinical and radiological assessment.

\section{CASE REPORT}

A 59-year-old male presented with a four-month history of headaches. Neurological examination revealed no focal deficits. Brain magnetic resonance imaging (MRI) showed a well- demarcated, solid mass in the left cerebellum of about $4.5 \times$ $3.1 \times 3.3 \mathrm{~cm}$ around the transverse and sigmoid sinus area. The mass demonstrated a heterogeneous low signal on T2weighted images and an iso-low signal on T1-weighted images with signal voids suspected to be a vascular structure (Fig. 1). The mass was observed to be compressing the 4 th ventricle and became strongly contrast enhanced on T1-weighted images. Preoperative angiography revealed tumor blush and tumor vessels coming off the branches of the anterior inferior cerebellar and posterior inferior cerebellar arteries. We performed a positron emission tomography (PET) to determine hypometabolism in the left cerebellum without metastatic lesion to other organs. Based on the clinical and radiological findings, we decided on a surgical procedure for therapeutic management. We performed a left suboccipital craniotomy for removal of the mass and pathological diagnosis. The tumor mass was of a pinkish, hard consistency. During the tumor removal, we experienced massive bleeding from inside the tumor. At the end stage of the tumor removal, we identified that the tumor base invaded the transverse and sigmoid sinuses. We at first tried to remove the invading portion, but were unable to do so for fear of massive bleeding. Histologically, the tumor cells were round to spindle-shaped, and branching vessels inside the tumor tissue showed the 'staghorn' appearance; and the immunohistochemical stain revealed that the tumor 

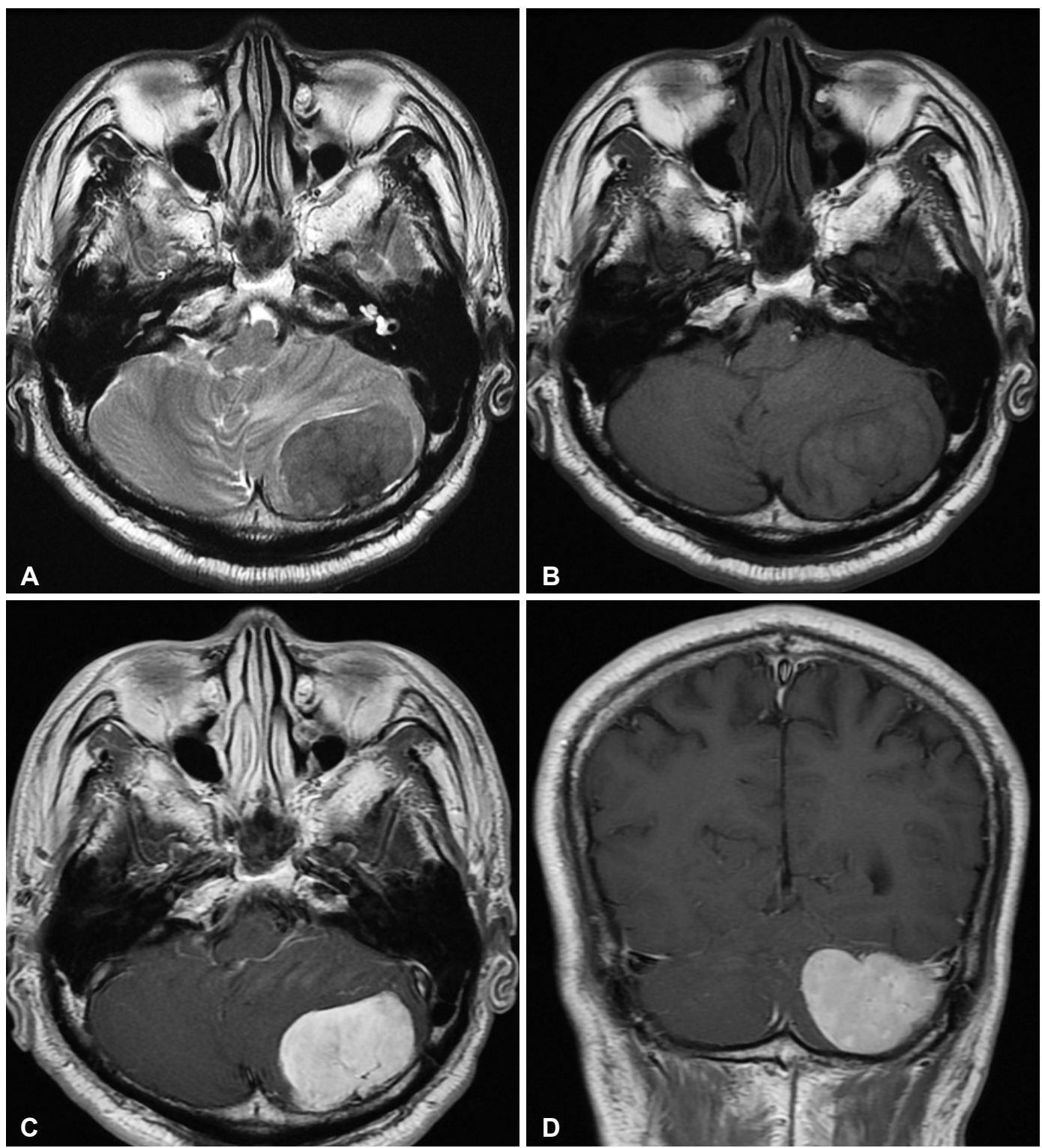

Fig. 1. Pre-operative axial $T 1$ (A) and T2 (B) magnetic resonance images show a tumor mass in the left cerebellum compressing the 4th ventricle with surrounding edema. Gd-enhanced T1 axial (C) and coronal (D) images reveal a well-enhancing lesion with dural attachment adjacent to the junction of the transverse and sigmoid sinuses.

cells were diffuse positive for CD-34, which was compatible with HPC [World Health Organization (WHO) grade II] (Fig. 2). Postoperative MRI performed two weeks after surgery showed a remarkable reduction in tumor size, with a residual mass adherent to the left transverse sinus (Fig. 3). Adjuvant radiotherapy with a total dose over 5400 cGy was administered to complete the treatment.

\section{DISCUSSION}

HPC is now widely accepted as an uncommon mesenchymal tumor that is believed to originate from Zimmerman's pericytes, which are contractile spindle cells surrounded with capillaries and postcapillary venules [3].

HPCs may occur anywhere in the body, usually in the musculoskeletal system and skin. The most commonly reported locations are the extremities, pelvis, and the head and neck areas [1]. Primary intracranial HPC has mostly been found in supratentorial regions and less frequently in the infratentorium [1]. Primary HPC located in the posterior fossa, such as in this case report has rarely been reported in neurosurgical literature since the new classification was established.

It has unique immunohistochemical characteristics in comparison to meningiomas, HPC was not classified as having an obvious individual categorization by the WHO until 1993 [3]. According to the current WHO classification 2007, HPC places 

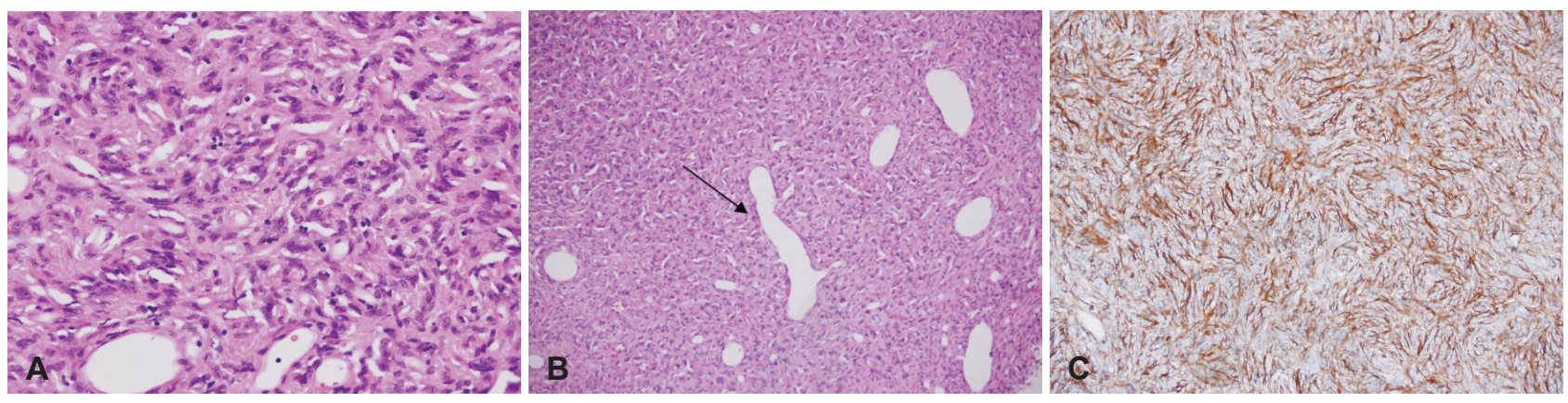

Fig. 2. A photomicrograph of the tumor specimen shows vascular space surrounded by proliferating oval to spindle-shaped pericytes (A) (hematoxylin and eosin stain; $\times 400$ ), prominent vasculature $(B)$ (hematoxylin and eosin stain; $\times 100)$ represented by staghorn pattern (arrow) and tumor cells strongly expressing CD-34 (C) (immunohistochemistry; $\times 200$ ).
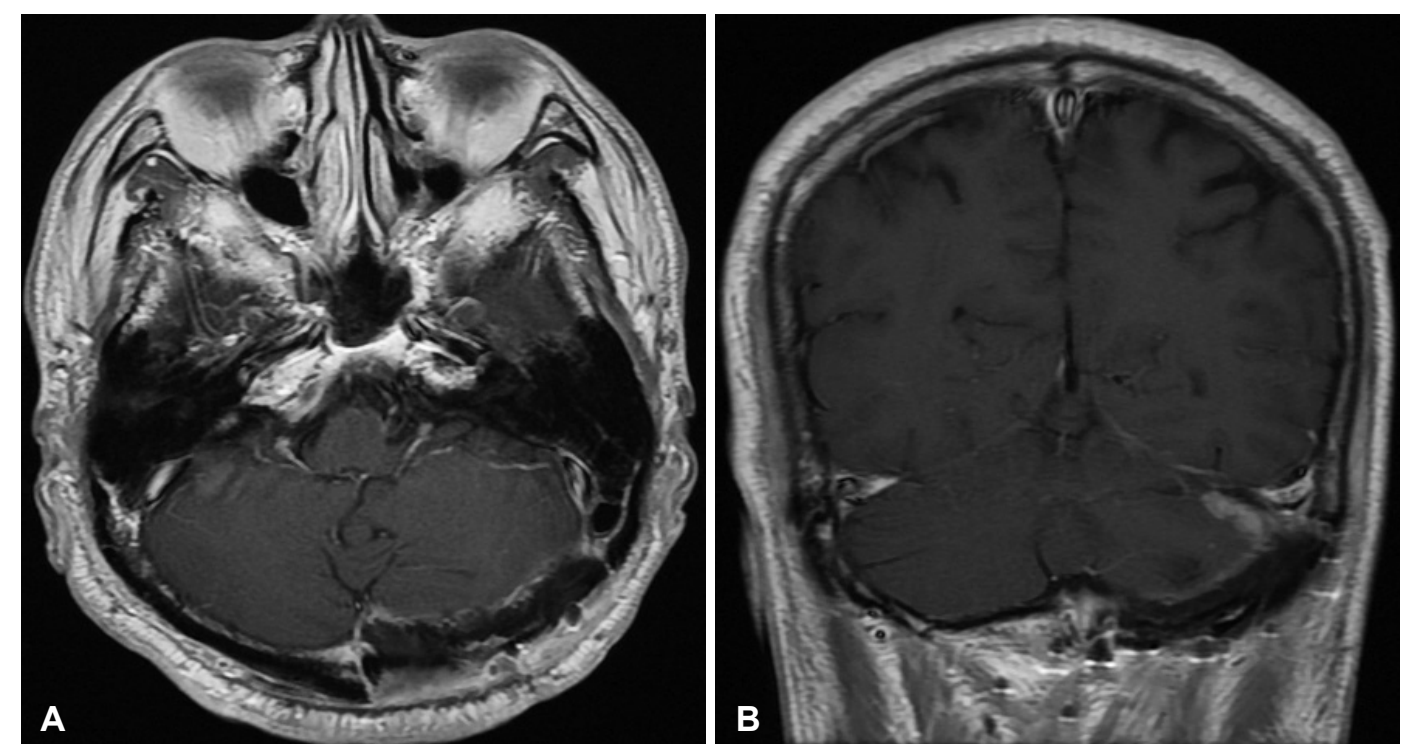

Fig. 3. Post-operative axial $T 1$ (A) and coronal $T 1$ (B) magnetic resonance images taken within two weeks of the surgery show the resection cavity with residual tumor tissue adherent to the transverse sinus.

itself as a subtype of "mesenchymal, non-meningothelial tumors" [4].

Reviewing other literatures, we found a study reporting primary HPC located in the posterior fossa and cerebello-pontine angle as $12 \%$ of intracranial HPCs (range, 5-21\%) [5-9]. The above authors did not distinguish clearly whether the HPCs were located in the posterior fossa or the cerebello-potine angle. When HPCs are restricted to the posterior fossa, the morbidity rate of HPC may be further decreased. HPC of the posterior fossa that invades the sinus, as in this case, has been very rarely published. Differentiation between HPCs and meningiomas is also very difficult based on radiological features and often poses a dilemma for neurosurgeons. HPCs usually presents as well-enhancing extra-axial lesions on MRI. This is not helpful enough to distinguish between the above two entities. Other tumors should also be considered in the differential diagnosis of tumors located in the posterior fossa, such as solitary fibrous tumors, angiosarcomas, chondrosarcomas, and metastatic carcinomas. Meningiomas are especially the most difficult to be differentiated from HPCs among other tumors based on the radiologic features. Several authors have reported that lytic destruction of the adjacent skull on plain X-rays or computed tomography (CT) suggests an HPC while hyperostosis suggests a meningioma [3]. HPC sometimes presents with multi-lobulated portions mimicking a mushroom appearance without calcifications [5]. Other studies that focused on dural attachment morphology suggested that dural attachment of HPCs may be seen narrower than that of a meningioma [5]. In this case, MRI showed a multi-lobulated (mushroom appearance), prominent internal signal voids, and relatively narrow dural attachment without calcifications. These findings highly suggested that the tumor was an HPC rather than a meningioma.

Some authors have found a significant difference in the mean minimum apparent diffusion coefficient values between HPCs and meningiomas; one reason for this may result from the pathological nature of the tumor, such as less dense cellularity and abundant vasculature in the tumor parenchyma [6]. 
Some authors have attempted to determine the usefulness of PET in the diagnosis of HPC. They exerted that HPC exhibited a significantly increased uptake of 11C-methionine, hyperperfusion, and glucose hypometabolism on PET imaging. These features may be helpful in distinguishing HPC from meningiomas [2]; however, PET involves a high risk of radiation exposure, limited accessibility not to mention the high cost. Recent studies have suggested that the proper role of PET in terms of HPC may be in identifying local recurrence and distant metastases after diagnosis of HPC [3].

Confusing and sometimes misleading, the above-mentioned imaging characteristics are not always crucial for diagnosing an HPC. For this reason, immunohistochemical findings should seriously be reviewed in the diagnosis of an HPC. Macroscopically, HPCs exhibit high vascularity, with intrinsic appearance of gray color in the visible vascular spaces. Microscopically, a prominent "staghorn" vascular channel around a reticulin network is a common finding with the tumor cells round to spindle-shaped. These findings differ from those of meningiomas, namely nuclear pseudo-inclusions and psammoma bodies [3]. Many of these tumors express a certain vascular or endothelial markers, including CD-34, as is true with our case, and Leu-7 [3].

Considering the aggressive nature of HPCs, gross total resection of the tumor is the optimal treating method $[3,8]$. Excision of involved dura and bone is required during the initial surgery; if this is not possible, mandatory aggressive cauterization of dura and bone should be the next scheme [7]. The local recurrence rate varies from $45 \%$ to $91 \%$ among different studies, and a 15-years distant metastasis rate approaches $70 \%$ after surgery alone [3]. Entailing postoperative radiotherapy is recommended in some reports to improve the disease-free survival rate. Although the responsiveness of HPC to radiotherapy is dose dependent; the dose of radiation is recommended to be at least 45 to $50 \mathrm{~Gy}[2,3]$.

Stereotactic radiosurgery may be an alternative option for the treatment of HPCs that are less than $8 \mathrm{~cm}^{3}(<2 \mathrm{~cm}$ in diameter) in volume, with radiation doses of $15 \mathrm{~Gy}$ or higher at the $50 \%$ isodose line [3]. Considering the fact that complete resection is difficult due to the high vascular and consequent bleeding tendency in the operative field, some authors have suggested the use of radiotherapy as a neoadjuvant treatment based on the possibility that the proliferating capillaries will be obliterated after radiation.

We have reported a rare case of HPC that arose in the posterior fossa around the transverse sinus. Correct diagnosis of HPCs can only be made through immunohistochemical study because of the clinical and radiological similarity between the HPCs and meningiomas. In particular, the unusual location of HPC often makes it difficult to diagnose via radiological study alone. Due to relatively high tendency of intra-operative bleeding of HPCs and recurrence even after gross total resection, the importance of differentiation between HPCs and meningiomas cannot be overemphasized. Preoperative correct diagnosis is difficult but it is important that it should be made. Multi-lobulated (mushroom appearance), prominent internal signal voids, relatively narrow dural attachment, and lytic destruction without calcifications are useful findings to distinguish HPCs from meningiomas.

\section{Conflicts of Interest}

The authors have no financial conflicts of interest.

\section{REFERENCES}

1. Kim JH, Kwon TH, Kim JH, Park YK, Chung YG, Chung HS. Meningeal hemangiopericytoma: study of 6 cases and review of the literatures. J Korean Neurosurg Soc 2006;39:32-5.

2. Fountas KN, Kapsalaki E, Kassam M, et al. Management of intracranial meningeal hemangiopericytomas: outcome and experience. Neurosurg Rev 2006;29:145-53.

3. Tashjian VS, Khanlou N, Vinters HV, Canalis RF, Becker DP. Hemangiopericytoma of the cerebellopontine angle: a case report and review of the literature. Surg Neurol 2009;72:290-5.

4. Louis DN, Ohgaki H, Wiestler OD, et al. The 2007 WHO classification of tumours of the central nervous system. Acta Neuropathol 2007;114: 97-109.

5. Chiechi MV, Smirniotopoulos JG, Mena H. Intracranial hemangiopericytomas: MR and CT features. AJNR Am J Neuroradiol 1996;17: 1365-71.

6. Liu G, Chen ZY, Ma L, Lou X, Li SJ, Wang YL. Intracranial hemangiopericytoma: MR imaging findings and diagnostic usefulness of minimum ADC values. J Magn Reson Imaging 2013. [Epub ahead of print]

7. Spatola C, Privitera G. Recurrent intracranial hemangiopericytoma with extracranial and unusual multiple metastases: case report and review of the literature. Tumori 2004;90:265-8.

8. Guthrie BL, Ebersold MJ, Scheithauer BW, Shaw EG. Meningeal hemangiopericytoma: histopathological features, treatment, and long-term follow-up of 44 cases. Neurosurgery 1989;25:514-22.

9. Alén JF, Lobato RD, Gómez PA, et al. Intracranial hemangiopericytoma: study of 12 cases. Acta Neurochir (Wien) 2001;143:575-86. 